\section{SERUM THERAPY WITH REPORT OF} CASES

Read before the Tri.State Medical Society, st. Louis, Mo., April 7, 1897 BY JOHN M. ALLEN, A.M., M.D.

PROFESSOR OF PRINCIPLES AND PRACTICE OF MEDICINE IN THE UNIVERSITY MEDICAL COLLEGE, KANSAS CITY, MO.

The announcement of the subject matter of my paper carries with it the idea that I would cover the field of serum therapy, but I shall confine myself to the use of antitoxic serum and its effects in diphtheria and phthisis pulmonalis.

The curative effects of antitoxic serum in diphtheria is now so well established that there is no need of argument or detail report of cases to establish its benefits in this disease to the medical profession. Hence I give only a summary of my cases. I have treated fifty-three cases of diphtheria without the loss by death of a single case. Forty of these cases were treated with serum only, cleanliness and good food. The remainder had had other treatment before $I$ saw them. The complications which occured among these cases were as follows: Hoarseness, seven; five of aphonia; two of toxemia; one of broncho-pneumonia resulting from the extention of the membrane into the bronchi; one case of paralysis. No operative interference was needed. In none of these cases of complication did I see them before the third day. All of the cases which I saw within the first twentyfour hours recovered without complication. Hence it is my opinion that cases seen thus early rarely have any complications.

My mode of administering the serum is to begin with a full dose the first day; two-thirds the next day; one-half the next day in mild cases. If the case is of malignant variety, I repeat full doses of serum every ten hours until improvement begins. Then lessen the dose, and give from two to four more. This repeated administration of the serum is based on the fact that the bacilli may be found in the fauces for several weeks after the membrane is thrown off.

It does occasionally happen that the larynx has been attacked a week or ten days after the membrane disappears from the fauces. But where the remedy is continued as directed above, the bacilli are either destroyed or are so attenuated as to lose their vital energy. It is my opinion that the serum has immunizing properties, because $\mathrm{I}$ injected it into seven children who were dangerously exposed to the disease. None of them had it. The dose used was full size.

I have treated four cases of phthisis pulmonalis with Paquin's antitoxic tuberculosis serum.

Case 1.-June 1894, Mrs. D., age 32, mother of one child, presented berself for treatment and I found her in the following condition: Hereditary tendency to phthisis, had gradually lost flesh and strength until reduced from 115 pounds to 75 pounds, in a period of about twelve months. Severe cough and expectoration for about last seven menths. Sputum had been examined about four months before I saw her and found to contain bacilli tuberculosis. At the time of my first visit she stated that she had had hectic fever and colliquative sweats daily for some time. Temperature 103.5 , respiration 37, pulse 98. Entire loss of appetite. Sputum under microscopic examination found to contain large quantity of bacilli. Auscultation and percussion located two spots in upper portion of right lung that were impervious to air. There was no evidence that the tissues had broken down.

I began the treatment with Paquin's antitoxic tuberculosis serum, giving ten drops by hypodermic injection, increasing the dose within three days to a dram, which was continued daily for 120 days. After which for another 120 days it was given every other day. After this it was given three or four times a week for the remainder of the year. This treatment was supplemented by the following prescriptions:
B. Creosote.
xxiv gtt.
130
Tr. cinnamom Glycerin

$\begin{array}{ll}3 \mathbf{3} & 16,00 \\ \mathbf{3 x} & 80,00\end{array}$

Sig. Teaspoonful gradually increased to five teaspoonful three imes daily before eating.

R. Oil eucalyptus. . . . . . . . 3i 400

Oil cassia

Creosote

Oil tar

400

400

Sig. Inhale in nebulizer frequently.

By the end of the third week her appetite began to return, hectic fever cessed, cough and expectoration gradually ceased and in less than six months she weighed 120 pounds. From the end of the third month the bacilli began to be attenuated and continued to decrease in size and number until the end of the fifteenth month when none could be found in the sputum nor has there been any since. The points of dulness in the lungs have greatly decreased in size, but have not entirely disappeared.

Case 2.-July 1895. Unmarried lady, age 26; hereditary tendency to phthisis. Had been an invalid for five years, vis. iting many climates in search of health. When presented to me I found her in the following conditions. Extreme emacia tion, loss of appetite, hectic fever, chronic diarrhea for the last fifteen months; pulse 110 , evening temperature 103.5, respirations 45 ; auscultation and percussion revealed a large abscess in apex of right lung. Mouth covered with aphthæ. Sputum loaded with bacilli. My prognosis was that she would not live sixty days. She was placed on the same course of treatment as Case 1. At the end of four weeks hectic fever had ceased. Her appetite had returned. Diarrhea very much better and entirely ceased before the sixth week. She steadily improved until the end of the fifth month, when a thrombus formed in the left femoral vein from which she died thirty days later.

Case 3.-December, 1895. There had existed for five years a tubercular deposit in the apex of the right lung. Childbirth caused it to rapidly break down. Microscopic examination showed tubercular bacilli. Began treatment and continued irregularly two months. This case was refractory to treatment from the beginning; therefore was not benefited and died at the end of five months.

Case 4.-March, 1896. Male, age 22, of good proportions. Had hemorrhages several times during the preceding year. Progressive emaciation ; weight 130 pounds, appetite not good. Evening temperature 101.5; respiration 34. Auscultation and percuesion indicated tuberculardeposits at two points of upper right lung. Microscopic examination of sputum showed an abundance of bacilli. Began treatment as above and within three weeks patient began to improve, which continued without interruption until he was compelled to leave me, September, 1896. At this time temperature normal, respirations 22 weight 150 , appetite good. Sputum contained attenuated bacilli. When last heard from he was at work on a ranch in Colorado.

In an analysis of the above cases it is fair to conclude that if this treatment was begun before the structures began to break down, it would be certainly beneficial and possibly curative. After abcesses have formed it is beneficial but probably not curative. Yet if the disease is not too far advanced in the second stage, many could be cured if supplemented by climate influences.

In estimating the curative value of the remedies used, the inhalation of germicidal remedies is certainly in the right direction; but alone they are useless. The creosote has for years had some reputation as a cure for phthisis. That it is beneficial, there is no question; but it is not curative, for the reason that it practically has no effect on the bacilli. This I have many times demonstrated by microscopic inspection of sputa in patients in my clinic at the University 
Medical College of Kansas City, who had taken large quantities of it. Creosote is beneficial in two ways. 1. It reduces the toxins in the blood, thereby lessening or preventing hectic fevers and colliquative sweats. 2. In a very large percentage of cases of phthisis pulmonalis next to the lungs the most frequent structure involved is the duodenum with chronic inflammation and ulceration. This interrupts intestinal digestion and assimilation, also often by reflex irritation interrupts stomach digestion. Hence is the progressive emaciation in phthisis. Creosote stands at the head of the list as a curative remedy in this pathologic condition, thereby increasing the digestive and assimilative force of the person.

With these facts before me I must conclude that the active force in affecting the bacilli was the serum. The next question is as to the effects of the serum after entering the blood. On this point many theories have been advanced.

I abstract the following from an address delivered by Dr. William Carter, of Philadelphia, before the Pan-American Medical Congress, 1893. "That blood serum is a powerful and active therapeutic force is just now beginning to be recognized. Landois discovered more than twenty years ago that the mixing of the blood of different animals resulted in the destruction of red corpuscles." Recently Dr. Carter by experiment has established the globucidal property of blood serum beyond question. $H_{\theta}$ has further demonstrated that the serum of some animals are destructive to the life of others. Mettal and Buckner have shown that the blood serum of certain animals which are refractive to certain diseases is highly destructive to the bacteria that caused the disease. This shows that there are other forces in the blood which are destructive to bacteria, besides that of Metchnikoff's theory of phagocytosis. It is also well established that animals naturally susceptible to certain diseases can be immuned to those diseases by injection of the blood serum of animals either naturally immune or rendered so artificially. That the injection of blood serum of immune animals after infection has taken place will destroy it and terminate this disease is true.

That serum has globucidal and germicidal properties is well established. Its globucidol effects can be well understood, but whether its germicidal effect be by actual contact or by destroying the entity upon which the germ lives and develops is doubtful.

In view of the fact that the life and development of the germ depends as much on its environments as that of the human family, and further that its life and development ceases as soon as the pabulum upon which it lives is consumed or destroyed, we deduct the idea that serum destroys this pabulum, thereby terminating the life of the germ. This is proven by the natural history of all the contagious diseases, first by their being self-limited, secondly by the fact that one attack in the majority of them immunes against a second, no matter how badly we may be exposed to the contagion. Indeed inoculation will not produce the disease. For no other reason than the entity upon which the germ lives and develops has been eradicated by previous attack. When serum is injected into the blood to immunize the person, it is done before there exists any germs in the blood; therefore, not for its germicidal effect. The only phenomena observed is a slight reaction attended with elevation of temperature. Hence immunity is nothing more than the eradication of this entity upon which this germ lives and develops. I give as an example, smallpox. After the person has been immuned from the disease no difference can be detected by the microscope or otherwise between the immune person from smallpox and the one who has not been. The blood, the secretion and exhalation are exactly alike. I think this holds good in all germ contagious diseases. We see this exemplified in the following process of fermentation, acetous, venous, alcoholic. The bacterial ferment lives and develops as long as the fluid element contains the essentials necessary for its life, after which it dies and no further germ life can exist in this fluid. The same is true of all culture media.

Hence it is my opinion that the curative effect of serum is indirectly germicidal by destroying an entity, which is necessary for the life and development of the bacilli. This is manifested in the fact that after its use the bacilli becomes attenuated; and from the further fact that many of us do not possess this entity. Hence the bacilli can not develop in our structures.

\section{A FEW NOTES ON SQUTNT BY WILLIAM B. MEANY, M.D. sT. LOUIS, Mo.}

It has long been known that hyperopes easily become the victims of exaggerated strabismus constituting squint; the disturbance between the two functionsaccommodation and convergence-leading to this result. The accommodation is measured and recorded by the Nagels plan of metric angles-a plan which corresponds with the metric measurement of the accommodation.

An emmetrope looking at an object half a meter away would use two diopters of accommodation; he would at the same time converge to the same point, and thus convergence would be expressed by two meter angles, so that we record it thus: $A=2 \mathrm{D}$; $\mathrm{C}=2 \mathrm{~m}$ a. If the object looked at be $20 \mathrm{~cm}$. off, then: $\mathrm{A}=5 \mathrm{D} ; \mathrm{C}=5 \mathrm{~m}$ a. The position of the p. p. (near point) and the p. r. (far point) of accom. modation vary according as the patient is emmetropic, hyperopic or myopic; and in all, the p. p. and p. r. of convergence is much the same, whatever the state of refraction may be, and the amplitude of convergence is not reduced by advanced age.

The foregoing remarks show how much latitude must be allowed when considering the connection between the linked functions of accommodation and convergence. Therefore the hyperope for a given amount of convergence will have to call into play an amount of accommodation in excess of his convergence equal to the amount of his hyperopia. Now if accommodation does not exceed the positive part of his range of accommodation, he will be able to see clearly; this is the case in the lower degrees of hyper. metropia, but in the higher degrees it will be impossible to harmonize these two functions which preside over binocular distinct vision. The amount of accommodation necessary for clear vision calls into action a greater amount of convergence than can be used for that particular distance, and convergent strabismus is the result.

I am aware that in thus considering the connection between accommodation and convergence, one is assuming that binocular fixation is a faculty which exists from birth, and not (as it might be considered) an acquired habit. 